\title{
Quantum computing and quantum measurement with mesoscopic Josephson junctions
}

\author{
D.V. Averin \\ Department of Physics and Astronomy, SUNY at Stony Brook, Stony Brook, NY 11794-3800
}

(February 1, 2008)

\begin{abstract}
Recent experimental demonstrations of quantum coherence of the charge and flux states of Josephson junctions show that the quantum Josephson dynamics can be used to develop scalable quantum logic circuits. In this work, I review the basic concepts of Josephson tunneling and Josephson-junction qubits, and discuss two problems of this tunneling motivated by quantum computing applications. One is the theory of photon-assisted resonant flux tunneling in SQUID systems used to demonstrate quantum coherence of flux. Another is the problem of quantum measurement of charge with the SET electrometer. It is shown that the SET electrometer at the Coulomb blockade threshold is the quantum-limited detector with energy sensitivity reaching $\hbar / \sqrt{3}$ in the resonant-tunneling regime.
\end{abstract}

\section{INTRODUCTION}

Remarkable ability of Bose condensates to create a single quantum state occupied with a macroscopically large number of particles leads to an unusual situation when a macroscopic object behaves as a pure quantum state of a simple quantum mechanical system. This behavior is in stark contrast to the usual situation when macroscopic objects behave purely classically and do not show any collective quantum effects despite the underlying quantum dynamics of their microscopic elements. The macroscopic quantum states of Bose condensates can exhibit non-trivial quantum dynamics associated with the fact that the number of particles in the condensate and the phase of its wavefunction are non-commuting quantum variablesila.

Macroscopic quantum phenomena arising from this dynamics are best studied in Josephson tunnel junctions, where the role of bosons is played by Cooper pairs of electrons, and both the number of pairs and the phase of the wavefunction give well-defined and controlled contributions to the junction energy. The "number" part of the energy comes from the Coulomb charging of the junction capacitance $C$ by Cooper pairs and has characteristic magnitude $E_{C} \equiv(2 e)^{2} / 2 C$, while the pair tunneling across the junction creates the "phase" term in the energy of magnitude $E_{J}$. The nature of the quantum dynamics of a Josephson junction depends on the ratio of these two characteristic energies. In large junctions with $E_{J} \gg E_{C}$ the phase behaves almost classically with quantum effects being "corrections" to semiclassical dynamics. One of the first quantum effects studied in this regime is the macroscopic quantum tunneling of the phase - see reviews in the collection 3 . In the opposite case of small junctions with $E_{J} \ll E_{C}$, the junction dynamics can be interpreted as semiclassical dynamics of charge on the capacitance $C$. Characteristic feature of the charge dynamics is correlated tunneling of individual Cooper pairs one br pne between superconducting electrodes of the system 1 .

Macroscopic character of the quantum effects in
Josephson junction dynamics makes them attractive as the basis for development of scalable quantum logic circuits for quantum computation. The regime of coherent quantum dynamics in Josephson tunneling can be reached in relatively large, micrometer-size junctions that can be designed and fabricated with large degree of precision. The relevant junction parameters, including the energies $E_{J}$ and $E_{C}$, reflect the collective properties of the superconductors forming the junction and are not sensitive to their precise microscopic structure on the atomic scale. This makes the Josephson junctions much less susceptible to the microscopic disorder unavoidable in the present-day fabrication technology, than, e.g., semiconductor quantum dots in the regime of coherent electron transport, also considered for quantum computing applicationst.

The advantage of scalability of Josepshon junction and, in general, solid-state qubits and logic circuits comes at a price of stronger dephasing and energy relaxation in these systems than in other non-solid-state qubits. Nevertheless, recent experimental demonstrations of quantum coherent Josephson dynamics both in chargel and phase 10 regime show that the problem of dephasing and energy relaxation in Josephson junctions is in principle solvable, and stimulate further studies of superconducting qubits. The aim of this work is to review the basic concepts of quantum dynamics of mesoscopic Josephson junctions and qubits, and to discuss two specific questions motivated by quantum computing applications of Josephson junctions: the theory of coherent effects in the photon-assisted resonant flux tunneling, and the problem of quantum measurement with SET electrometer.

\section{JOSEPHSON TUNNELING IN MESOSCOPIC JOSEPHSON JUNCTIONS}

An isolated mesoscopic Josephson tunnel junction is a system of two small superconducting islands separated by an insulator layer that is sufficiently thin to allow electrons to tunnel between the islands. The junction 
is "mesoscopic" if its capacitance $C$ is small enough for the charging energy $E_{C}=(2 e)^{2} / 2 C$ of a single Cooper pair to be sufficiently large. Quantitative description of this system is most transparent when the superconducting energy gap of the islands is much larger than other characteristic junction energies, including charging energy $E_{C}$ and temperature $T$. In this case, quasiparticles can not be excited in the superconductors and the junction properties can be understood simply from the standard notion that the superconductors are Bose-Einstein condensates of Cooper pairs, with Cooper pairs occupying a single quantum state in each island. If $n_{1}$ and $n_{2}$ are the numbers of Cooper pairs in the two superconducting islands, the imbalance $n$ of the number of Cooper pairs between the two superconductors, $n=\left(n_{1}-n_{2}\right) / 2$, gives the charge $2 e n$ on the junction capacitance $C$. The junction Hamiltonian depends on $n$ through the charging energy $(2 e n)^{2} / 2 C-2 e n V_{e}$, where $V_{e}$ is the potential difference induced between the two superconducting islands by external electric field.

Another part of the Hamiltonian comes from the tunneling of Cooper pairs between the islands. In accordance with the standard practice, we denote the amplitude of this tunneling as $-E_{J} / 2$ and take into account that the transfer of a Cooper pair between the islands changes $n$ by \pm 1 . Then, combining the electrostatic and the tunneling terms, we get the total Hamiltonian $H$ of the junction in the $n$-representation:

$$
H=E_{C}(n-q)^{2}-\frac{E_{J}}{2}(|n\rangle\langle n+1|+| n+1\rangle\langle n|) .
$$

Here $q \equiv C V_{e} / 2 e$ is the charge (in units of $2 e$ ) induced on the junction capacitance by the source of potential $V_{e}$. The energy $E_{J}$ of Cooper pair tunneling is usually referred to as Josephson coupling energy. It can be expressed in terms of the normal-state tunnel resistance of the junction.

Behavior of a single Josephson junction described by the Hamiltonian (11) is non-trivial due to important difference between the two charges $n$ and $q$ that define its charging energy. The charge $n$ originates from the total number of Cooper pairs in a superconducting island and can take on only integer values, as a charge of any isolated conductor. By contrast, the charge $q$ is the polarization charge induced on the capacitance $C$ by external electric field and can be varied continuously. Because of this difference, ejgenstates of the Hamiltonian (11) form energy bands 11 periodic in $q$ with the period one. When $q$ changes by one, a Cooper pair is transferred through the junction increasing $n$ also by one, and fully compensating for the increase of $q$, whereas smaller changes of $q$ can not be compensated by any transfer of integer number of Cooper pairs.

An example of the periodic dependence of junction properties on $q$ is given in Fig. 17a which shows one period of $q$-dependence of the ground state energy $\varepsilon_{0}$ of the junction obtained by direct numerical diagonalization of the Hamiltonian (11) for several values of the $E_{J} / E_{C}$ ratio. Periodic oscillations of the ground-state energy, and similar oscillations of energies $\varepsilon_{m}$ of the excited states, result in oscillations of all other junction characteristics with $q$. For instance, differentiation of the junction free energy $F=-T \ln \sum_{m} e^{-\varepsilon_{m} / T}$, with repect to $q$ gives the average number of Cooper pairs $\langle n\rangle$ charging the junction:

$$
\langle n\rangle=q-\frac{1}{2 E_{C}} \frac{\partial F}{\partial q},
$$

and the average voltage across the junction $V(q)=$ $2 e(\langle n\rangle-q) / C$.
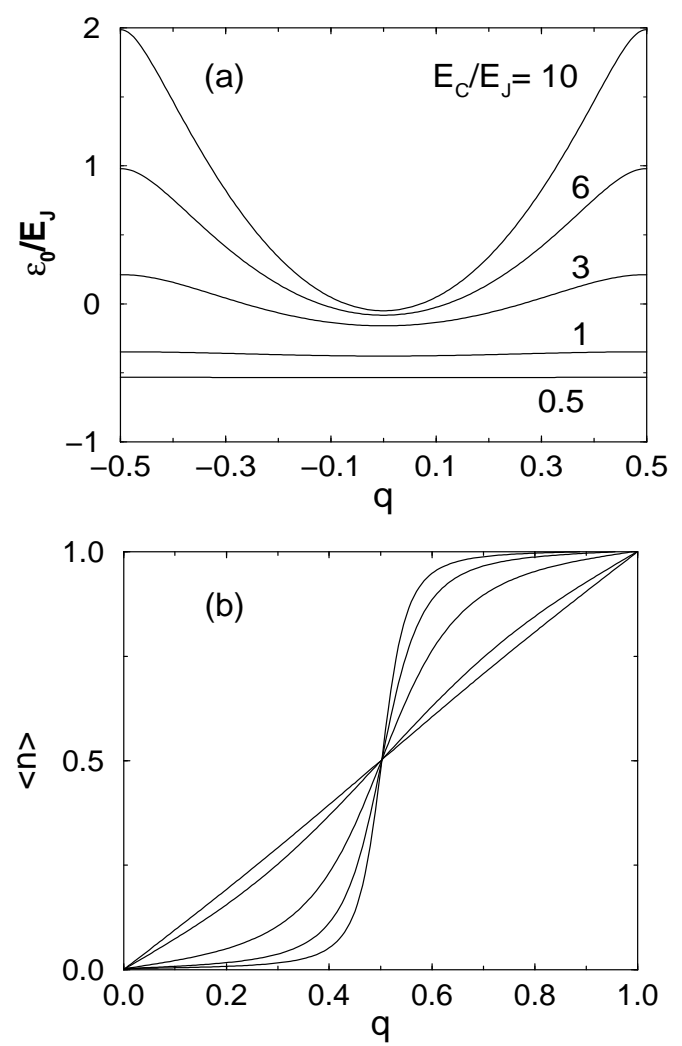

FIG. 1. The ground state energy (a) and the average number of Cooper pairs on the junction capacitance (b) for an isolated mesoscopic Josephson junction, as functions of the external charge $q$ injected into the junction. The curves in (b) are for the same values of the ratio $E_{C} / E_{J}$ as in (a).

Average number of Cooper pairs (2) on the junction capacitance as a function of $q$ is shown in Fig. 1 $1 \mathrm{~b}$ for zero temperature and different $E_{J} / E_{C}$ ratios. In the limit of small coupling energies $E_{J}$, the number of pairs $\langle n\rangle$ increases in a step-wise manner remaining constant for almost all of $q$ values exept for half-integer $q$ 's, when it rapidly increases by one. Qualitatively, at $E_{C} \gg E_{J}$, the supercurrent flow between the junction electrodes is "discretized" into the transfer of individual Cooper pairs by strong Coulomb repulsion, and variations of the charge $q$ injected into the junction leads to the possibility of 
controlling their tunneling.

Step-like behavior of $\langle n\rangle$ at $E_{J} \ll E_{C}$ reflects quantization of the number of Cooper pairs in the superconducting electrodes of the junction. When $E_{J}$ increases, the junction electrodes become strongly coupled and the quantization of $n$ is gradually washed out. In this case, the ground state energy of the junction (Fig. 17a) becomes virtually independent of $q$, and the average number $\langle n\rangle$ of Cooper pairs on the junction follows closely the induced charge $q$ (Fig. 1 $1 \mathrm{~b}$ ), $\langle n\rangle \simeq q$. Qualitatively, this suppression of $n$ quantization with increasing $E_{J}$ is the result of large quantum fluctuations of $n$ induced by tunneling terms in the Hamiltonian (11).

At $E_{J} \geq E_{C}$, the junction properties can be described more directly by introducing the phase operator $\varphi$ canonically conjugated to the number $n$ of Cooper pairs. From quantum mechanics of a single boson model, it is known that the operator of the phase $\varphi_{j}$ of the condensate wavefunction in one of the junction electrodes, $j=1,2$, can be introduced through the operators $s^{ \pm}=|n \pm 1\rangle\langle n|$ (omitting the index $j$ to simplify the notations) raising and lowering the number of Cooper pairs in the condensate. On all of the states $|n\rangle$ besides the state $|0\rangle$ with no particles, the operators $s^{ \pm}$satisfy the relations $s^{+} s^{-}=s^{-} s^{+}=1$ and $s^{-}=\left(s^{+}\right)^{\dagger}$ which suggest that they can be expressed as

$$
s^{ \pm}=e^{ \pm i \varphi} .
$$

In fact, since the state $|0\rangle$ is annihilated by $s^{-}, s^{-}|0\rangle=0$, the commutator of $s^{+}$and $s^{-}$does not vanish:

$$
\left[s^{-}, s^{+}\right]=|0\rangle\langle 0| .
$$

Non-vanishing commutator contradicts the possibility of expressing the operators $s^{ \pm}$through one phase operator, and leads to the well-known problem with a generic definition of the phase of a boson mode, discussed in the literature on quantum optics - see, e.g., the review 12 and references therein.

However, in the case of a Bose condensate with macroscopically large number of particles, all relevant states do not have the component $|0\rangle$, the commutator (4) effectively vanishes, and eqs. (3) define the operators $e^{ \pm i \varphi}$. Then, the commutator between $n$ and the raising/lowering operators can be written as

$$
e^{ \pm i \varphi}(n \pm 1)=n e^{ \pm i \varphi} .
$$

For a superconducting island, the number of Cooper pairs in the condensate can be estimated as the ratio $\Delta / \delta$ of the superconducting energy gap $\Delta$ over the spacing $\delta$ of the single-particle energy levels. Condition $\Delta \gg \delta$ necessary for defining the phase operator coipcides with the criterion of the BCS superconductivity 13,4 .

With the raising and lowering operators $e^{ \pm i \varphi_{j}}$ for the two condensates in the junction electrodes, the operator of transfer of a Cooper pair through the junction can be written as $e^{ \pm i \varphi} \equiv e^{ \pm i\left(\varphi_{1}-\varphi_{2}\right)}$. They have the same commutators (5) with the number of Cooper pairs $n$ on the junction capacitance $C$. The operators $e^{ \pm i \varphi}$ allow one to define any periodic function of $\varphi$, and the commutation relations (5) imply that in this periodic $\varphi$ representation, the number of Cooper piars can be written as $n=-i \partial / \partial \varphi$. (The aperiodic operator of the phase $\varphi$ itself can not be defined for an isolated junction, since $n$ is integer and all junction properties should be periodic in $\varphi$ with the period $2 \pi$ ). The Hamiltonian (11) is equivalent then to that of a plain rotor in an external field:

$$
H=E_{C}\left(\frac{1}{i} \frac{\partial}{\partial \varphi}-q\right)^{2}-E_{J} \cos \varphi .
$$

This representation of the Hamiltonian (11) allows one to analyze simply the behavior of the ground state energy $\epsilon_{0}$ and average charge $\langle n\rangle$ (Fig. 位) for $E_{J} \geq E_{C}$ in terms of tunneling through the coupling potential $-E_{J} \cos \varphi$.

Descrpition in terms of the phase dynamics becomes particular relevant in the situation of an "rf SQUID" (see Fig. 6 below), when the junction is included in external superconducting loop. Due to qmantization of the magnetic flux $\Phi$ in the loop (see, e.g., 15 ) the phase $\varphi$ is linked to the flux, $\varphi=2 \pi \Phi / \Phi_{0}$, where $\Phi_{0}=\pi \hbar / e$ is the "flux quantum". The states of this system that differ in phase by $2 \pi$ are clearly distinguishable, and the charge $n=-i \partial / \partial \varphi$ is no loger integer. The transition from periodic to aperiodic phase $\varphi$ in the Josephson junctions has been debated extensively in the earlydiscussions of charging effects in Josephson junctions 1116 18. In physical terms, in the rf SQUID, both electrodes of the junction are parts of the same conductor. In this situation, the charge $n$ is the polarization charge and can vary continuously. One consequence of this is that any external charge $q$ can be screened by charge transfer through the loop, so that $q$ does not have any effect on the junction dynamics and can be omitted from the Hamiltonian. Then, the Hamiltonian of the system, including the magnetic energy of the loop inductance $L$ threaded by an external flux $\Phi_{e}$ is:

$$
H=E_{C} n^{2}-E_{J} \cos \varphi+\frac{\left(\Phi_{0} \varphi / 2 \pi-\Phi_{e}\right)^{2}}{2 L} .
$$

In such an "aperiodic" system, the phase operator $\varphi$ is well-defined and has the standard commutation relation with the charge $n: 2$

$$
[\varphi, n]=i .
$$

Both the controlled quantum dynamics of individual Cooper pairs governed by the Hamiltonian (11) and quantum dynamics of flux (耳) can be used to build qubits for quantum computation. The main obstacle to this is dephasing and relaxation mechanisms present in the Josephson junction circuits that couple charge or flux motion to other degrees of freedom not included in eqs. (11) or (7). Such a coupling makes dynamics irreversible 
and suppresses quantum correlations of the charge or flux states. Although suppression of the relaxation strength to acceptably low level is a challenging problem, reversible quantum coherent dynamics has been demonstrated both in the charge and flux regimes In the charge case, this has been done implicitly 1921 , through observation of splitting of the two lowest energy eigenstates of the Hamiltonian (1) at $q \simeq 1 / 2$, and explicitly, by direct measurement of the time-dependent oscillations of a Cooper pairl. Quantum coherence of the flux states remained olysive for many years. Very recently, two experiments 10 reported first observations of the coherent superposition of the macroscopically distinct flux state, with superposition of states evidenced indirectly by the coherent splitting of the energy eigenstates of the Hamiltonian similar to (7). The splitting is caused by tunneling through the maximum of the SQUID potential $U(\Phi)$ for the flux dynamics. In the next section, I discuss briefly the theory of flux tunneling in one of these experiments. More detailed presentation of this theory can be found ine2.

\section{QUANTUM COHERENCE OF FLUX STATES}

With the appropriate choice of parameters of the SQUID Hamiltonian (7), $\Phi_{e} \simeq \Phi_{0} / 2$ and $E_{J} \simeq \Phi_{0}^{2} / 2 \pi^{2} L$, the SQUID potential $U(\Phi)$ has two minima separated by a potential barrier. Quantum coherence of flux state should manifest itself as coherent oscillations of flux between the two potential wells due to tunneling through the barrier. The evidence for such coherent oscillations was obtained in by studying photon-assisted resonant tunneling of flux between the two well. The energy diagram of this process is shown in Fig. 2.23 An rf signal of frequency $\omega$ resonantly couples the ground state $|0\rangle$ in the left well of the potential to an excited state $|1\rangle$ with energy $E$ in this well. The condition of the resonant excitation is that the detuning $\nu=E-\omega$ is small, $\nu \ll E$. If the amplitude $a$ of the rf excitation is also relatively small, $a \ll E$, photon-induced coupling between the states $|0\rangle$ and $|1\rangle$ can be described in the rotatingwave approximation, in which the terms in the coupling Hamiltonian that oscillate rapidly in time are neglected. The excited state $|1\rangle$ in the left well is coupled resonantly with amplitude $-\Delta / 2$ to a state $|2\rangle$ in the right well that is shifted in energy by $\varepsilon$ with respect to $|1\rangle$. The total Hamiltonian for the flux dynamics in the basis of the three states $|0\rangle,|1\rangle$, and $|2\rangle$ is:

$$
H=\left(\begin{array}{ccc}
0 & a / 2 & 0 \\
a / 2 & \nu & -\Delta / 2 \\
0 & -\Delta / 2 & \nu-\varepsilon
\end{array}\right) .
$$

The rate $\tau^{-1}$ of resonant flux tunneling between the wells of the SQUID potential in absence of rf signalet 26 is known to be qualitatively independent of the strength of the energy relaxation in the flux dynamics. The shape of the resonant tunneling peaks is the same both in the regimes of coherent and incoherent tunneling. In contrast to this, the rate of the photon-assisted tunneling is sensitive to the relaxation strength, with the resonant peaks acquiring additional structure in the regime of coherent tunneling, when the energy relaxation is weak.

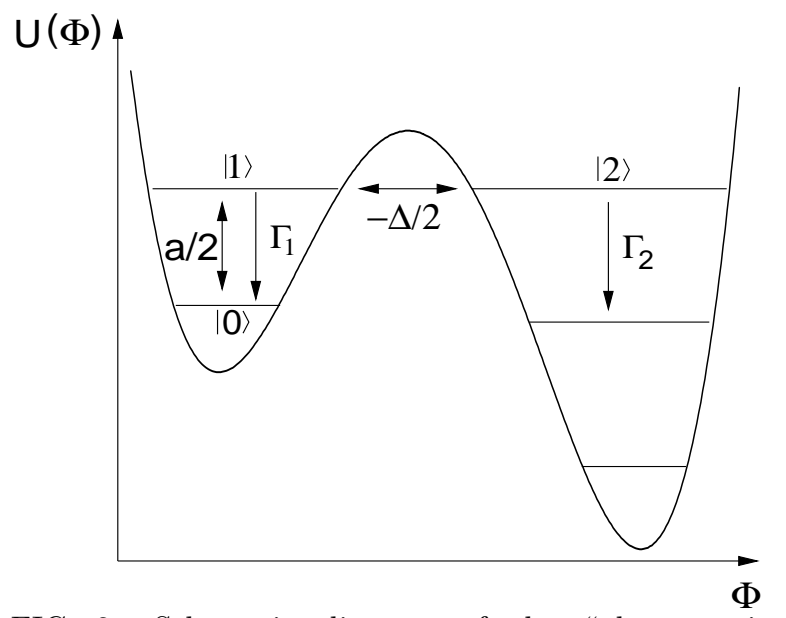

FIG. 2. Schematic diagram of the "photon-assisted" macroscopic resonant tunneling of flux stimulated by an $\mathrm{rf}$ perturbation of strength $a / 2$.

The main source of the energy relaxation for quantum dynamics of Josephson junctions is electromagnetic environment of the junction. Under the assumption that electromagnetic modes of the environment are in equilibrium at temperature $T$, the interaction between the flux $\Phi$ and the heat bath of these modes can be written as

$$
V=-I_{f} \Phi,
$$

where $I_{f}$ is the fluctuating current created in the SQUID loop by the environment, with the correlation function given by the fluctuation-dissipation theorem:

$$
\left\langle I_{f}(t) I_{f}(t+\tau)\right\rangle=\int \frac{d \omega}{\pi} \frac{\omega G(\omega) e^{i \omega \tau}}{1-e^{-\omega / T}} .
$$

Here the brackets $\langle\ldots\rangle$ denote averaging over the equilibrium density matrix of the environment, and $G(\omega)$ is the dissipative part of the environment conductance.

The coupling (10) leads to the two types of relaxation processes for dynamics of flux tunneling in the doublewell potential. One is the "intrawell" relaxation inducing transitions from the states $|1\rangle$ and $|2\rangle$ to the states with lower energies within the same well. In these transitions, the environment is absorbing large energy quanta on the order of energy separation between the states in the wells. Another is "interwell" relaxation which arises from the fluctuating energy shifts of one well relative to another caused by the coupling to environment (10). Interwell transitions transfer to the environment much smaller energy on the order of energies in the Hamiltonian (9).

The dissipative dynamics of flux with the Hamiltonian (9) can be described with the time-evolution equations 
for the density matrix $\rho$ of the system. These equations can be obtained in the standard way from the environment characteristics (10), (11) in the case of weak relaxation - see, e.g.27. Under the natural assumption that the average flux in the states $|0\rangle$ and $|1\rangle$ in the left well of the SQUID potential is the same, the part of the coupling (10) that corresponds to the interwell relaxation is:

$$
V=-I_{f} \delta \Phi\left(\begin{array}{ccc}
1 & 0 & 0 \\
0 & 1 & 0 \\
0 & 0 & -1
\end{array}\right) \equiv-I_{f} \delta \Phi U
$$

where $\delta \Phi$ is half of the difference between the average flux in the left and right wells. While eq. (12) is written in the flux basis $|0\rangle,|1\rangle,|2\rangle$, weak relaxation is conveniently described in the basis of the eigenstates $|n\rangle$ of the Hamiltonian (9). In this basis, the contribution of the weak interwell relaxation (12) to the evolution of the density matrix $\rho$ is given by the standard expressions:

$$
\begin{aligned}
\dot{\rho}_{n n} & =\sum_{m}\left(\gamma_{m n} \rho_{m m}-\gamma_{n m} \rho_{n n}\right) \\
\dot{\rho}_{n m} & =-\left[\gamma_{m n}^{\prime}+\frac{1}{2} \sum_{k}\left(\gamma_{n k}+\gamma_{m k}\right)\right] \rho_{n m}, \quad n \neq m
\end{aligned}
$$

Transition and dephasing rates in these equations are:

$$
\gamma_{n m}=\frac{g\left|U_{n m}\right|^{2}\left(\varepsilon_{n}-\varepsilon_{m}\right)}{1-e^{-\left(\varepsilon_{n}-\varepsilon_{m}\right) / T}}, \quad \gamma_{n m}^{\prime}=\frac{g T}{2}\left(U_{n n}-U_{m m}\right)^{2}
$$

where $U_{n m}$ are the matrix elements of the operator $U$ (12) in the eigenstates basis, $\varepsilon_{n}$ are the eigenenergies, and dimensionless parameter $g=2 G(\delta \Phi)^{2} / \hbar$ characterizes the strength of the interwell relaxation.

The intrawell relaxation has simple form directly in the flux basis. Equations for the off-diagonal elements of $\rho$ in this basis are:

$$
\begin{aligned}
& \dot{\rho}_{01}=\left(i \nu-\Gamma_{1} / 2\right) \rho_{01}+i a\left(\rho_{00}-\rho_{11}\right) / 2-i \Delta \rho_{02} / 2, \\
& \dot{\rho}_{12}=-\left(i \varepsilon+\left(\Gamma_{1}+\Gamma_{2}\right) / 2\right) \rho_{12}+i \Delta\left(\rho_{22}-\rho_{11}\right) / 2-i a \rho_{02} / 2, \\
& \dot{\rho}_{02}=\left(i(\nu-\varepsilon)-\Gamma_{2} / 2\right) \rho_{02}-i a \rho_{12} / 2-i \Delta \rho_{01} / 2 .
\end{aligned}
$$

The rates $\Gamma_{1,2}$ in these equations describe, respectively, the relaxation out of the states $|1\rangle$ and $|2\rangle$ into the lowerenergy states within the left and right wells. Diagonal part of the evolution equations is:

$$
\begin{aligned}
& \dot{\rho}_{00}=-a \operatorname{Im} \rho_{01}+\Gamma_{1} \rho_{11}+\Gamma_{2} \rho_{22}, \\
& \dot{\rho}_{11}=a \operatorname{Im} \rho_{01}+\Delta \operatorname{Im} \rho_{12}-\Gamma_{1} \rho_{11}, \\
& \dot{\rho}_{22}=-\Delta \operatorname{Im} \rho_{12}-\Gamma_{2} \rho_{22} .
\end{aligned}
$$

Equation for $\rho_{00}$ here was written under the assumption that after the relaxation in the right well the system is returned instantly to the initial state $|0\rangle$. This modification of the evolution equations allows us to calculate the rate $\tau^{-1}$ of the flux tunneling between the two wells from the stationary solution $\rho^{(0)}$ of the evolution equations 22 :

$$
\tau^{-1}=\Gamma_{2} \rho_{22}^{(0)} .
$$

The evolution equations for $\rho$ that include both the interwell and intrawell relaxation can be solved numerically. To do this, one diagonalizes the Hamiltonian (9), calculates the interwell relaxation terms (13) in the eigenstates basis and transforms them into the flux basis where the the intrawell relaxation has the simple form (14), (15). Finally, the stationary value of the density matrix $\rho$ gives the flux tunneling rate (16).

Figures 3 shows an example of the results of such a calculation obtained for vanishing temperature $T$ and $\Gamma_{1}=\Gamma_{2} \equiv \Gamma$. The tunneling rate is plotted as a function of the detuning $\nu$ for $\varepsilon=0$ and several values of the relative strength of the intrawell relaxation $\Gamma$. We see that the main resonant tunneling peaks is split in the two peaks due to coherent oscillations of flux between the two wells. Appearance of such splitting can be easily understood, since sufficiently weak rf signal excites the system not into the state $|1\rangle$ localized in the left well, but into the two hybridized states formed out of the states $|1\rangle$ and $|2\rangle$ in the two wells. The two different energies of the two hybridized states lead to the two peaks in the tunneling rate. In the time-domain, hybridization of the states $|1\rangle$ and $|2\rangle$ corresponds to the coherent oscillations of flux between these states. This means that the splitting of the resonant tunneling peak is the direct manifestation of the quantum coherent oscillations of flux between the two wells of the SQUID potential.

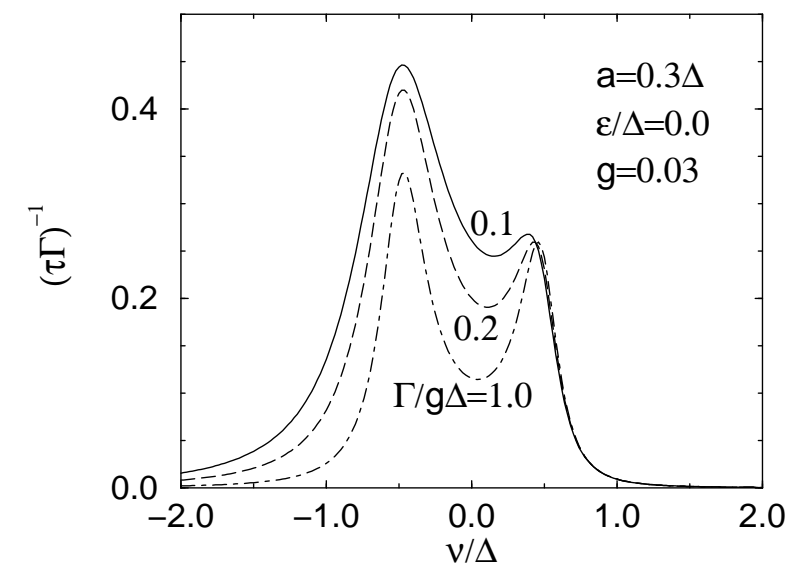

FIG. 3. The rate $\tau^{-1}$ of the photon assisted flux tunneling as a function of the detuning $\nu$ in the case of symmetric coupling between the tunneling flux states, $\varepsilon=0$, in presence of both the interwell and intrawell relaxation. Different curves correspond to different magnitude of the intrawell relaxation rate $\Gamma$ relative to the interwell relaxation.

At $\varepsilon=0$, the hybridized states are distributed symmetrically between the two wells, and asymmetry of the two peaks in Fig. 3 is caused by the interwell relaxation. The positive- $\nu$ side of the double-peak structure corresponds to excitation of the system into the lower-energy eigenstate and is unaffected by the interwell relaxation at zero temperature, since there is no energy in this 
regime to create additional tunneling path. In contrast, the negative- $\nu$ side of the double-peak structure corresponds to excitation of the system into the eigenstate with larger energy, and the interwell relaxation increases the rate of tunneling out of this state. Because of this, the negative- $\nu$ peak in Fig. 3 is larger that the tunneling peak at positive $\nu$, and the tunneling rate at $\nu<0$ decreases much more slowly away from the peak than at positive $\nu$.

In absence of the interwell relaxation, the double-peak structure is symmetric for $\varepsilon=0$ as illustrated in Fig. 4. This figure also shows how the splitting of the tunneling peak is suppressed with increasing relaxation strength. The curves are obtained by finding the stationary solution of eqs. (14) and (15) which are valid for arbitrary strength of the intrawell relaxation, $\Gamma_{1.2} \sim \Delta, a$, and neglecting the weak interwell relaxation (13) in this regime. They show that for $\Gamma \geq \Delta$ the two peaks of the doublepeak structure in the flux tunneling rate are suppressed and merge into one broad resonant tunneling peak.

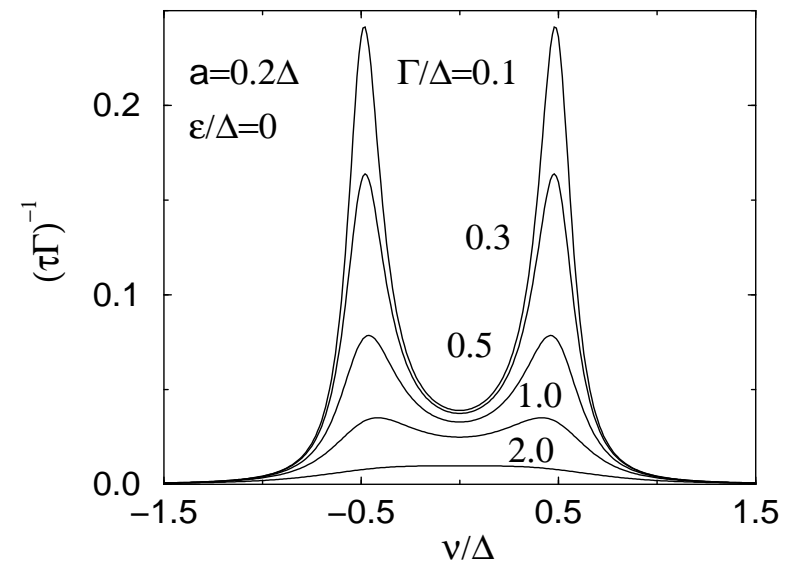

FIG. 4. Evolution of the double-peak structure in the rate $\tau^{-1}$ of the photon-assisted resonant flux tunneling as a function of detuning $\nu$ with increasing relaxation rate $\Gamma$.

It should be noted that for sufficiently large bias $\varepsilon$, the two peaks of the resonant flux tunneling exist even for strong relaxation rate $\Gamma$, as can be seen analytically in the limit $\Gamma_{1,2} \gg \Delta, a$. Expressing the off-diagonal element of $\rho$ in terms of the diagonal ones in the stationary regime from eqs. 14 and using their stationary values in eqs. (15) we find the stationary probability $\rho_{22}^{(0)}$ and the flux tunneling rate (16). For strong relaxation,

$$
\tau^{-1}=\frac{\Gamma_{2} a^{2} \Delta^{2}}{\left(4 \nu^{2}+\Gamma_{1}^{2}\right)\left(4(\nu-\varepsilon)^{2}+\Gamma_{2}^{2}\right)} .
$$

At large bias energy $\varepsilon$, the tunneling rate (17) as a function of detuning $\nu$ contains two separate peaks that correspond to excitation of the flux into the energy states $|1,2\rangle$ localized in the two wells. The two peaks merge, however, with decreasing bias and form one broad peak at $\varepsilon \simeq 0$ shown in Fig. 4 This behavior should be con- trasted with the coherent-tunneling regime with small $\Gamma$, when the two peaks remain separated even at $\varepsilon \simeq 0$.

In the experiment 9 , the flux tunneling rate $\tau^{-1}$ was studied as a function of the external flux $\Phi_{e}$ through the SQUID loop. Variations of $\Phi_{e}$ change both the energy shift $\varepsilon$ between the two wells of the SQUID potential $U(\Phi)$ and the separation $E$ between the levels within the same well. For a fixed frequency $\omega$ of external rf radiation, detuning $\nu=E-\omega$ changes together with $E$. Although there are some minor quantitative differences between the dependence of the tunneling rate $\tau^{-1}$ on $\Phi_{e}$ (which controls both $\nu$ and $\varepsilon$ ), and dependence of $\tau^{-1}$ on the detuning $\nu$ plotted in Figs. 3 and 1 , both dependences show the splitting of the resonant tunneling peaks due to coherent superposition of the flux states in the wells of the potential. This means that observation 9 of the splitting of the resonant peaks of the photon-assisted flux tunneling under the resonant bias conditions $\varepsilon \simeq 0$ demonstrates quantum coherent flux dynamics and quantum superposition of macroscopically distinct flux states. This coherent flux dynamics can be used for development of the flux qubits.

\section{CHARGE AND FLUX QUBITS}

Josephson junction qubits and quantum logic circuits can operate in the charge and flux regimes. To create a qubit, one needs to reduce the junction dynamics to two states and to be able to control accurately the resulting two-state dynamics. The most basic arrangement that satisfy these requirements in the charge space is the single-Cooper-pair box $28-30$ configuration, in which the external charge $q$ is injected into the junction by a gate voltage through an additional capacitance (Fig. 5a).

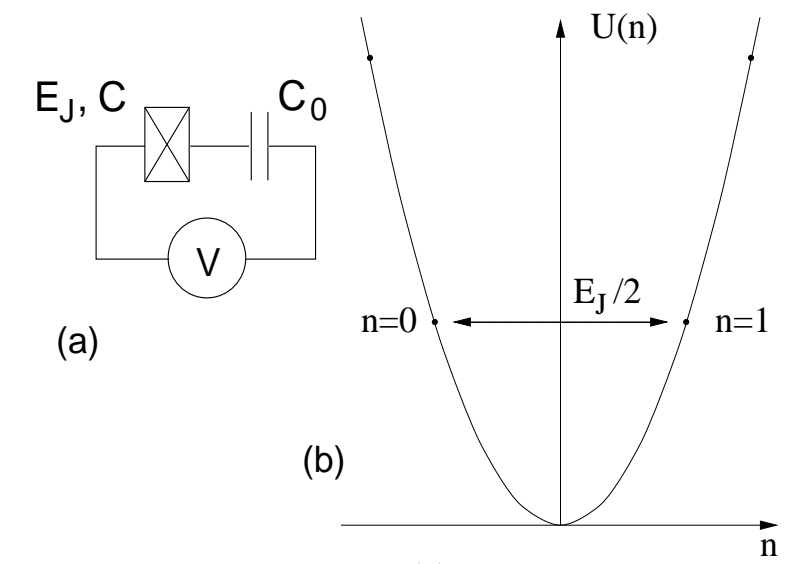

FIG. 5. Equivalent circuit (a) and the energy diagram (b) of the charge qubit. External gate voltage $V$ coupled through a small capacitance $C_{0}$ induces charge close to half a Cooper pair charge $2 e$ on the Josephson junction with coupling energy $E_{J}$ and capacitance $C$.

The charge qubit is the Cooper-pair box with small Josephson coupling energy $E_{J} \ll E_{C}$ and induced charge 
$q \simeq 1 / 2.3132$ In this case, the two charge states, $n=0$ and $n=1$, are nearly-degenerate and are separated from all other states by the large energy gaps on the order of $E_{C}$ (Fig. $5 \mathrm{~b}$ ). The two states are coupled by the tunneling of one Cooper pair, and the junction Hamiltonian (1) is effectively reduced to the two-state Hamiltonian:

$$
H=E_{C}\left(q-\frac{1}{2}\right)(|0\rangle\langle 0|-| 1\rangle\langle 1|)-\frac{E_{J}}{2}(|0\rangle\langle 1|+| 1\rangle\langle 0|) .
$$

The energy difference between the charge states is controlled through $q$ by the gate voltage, while their tunnel coupling can be controlled by the external magnetic field that modulates the Josephson coupling energy $E_{J}$. Operation of a Cooper-pair box as a qubit has been demonstrated experimentally in 8 .

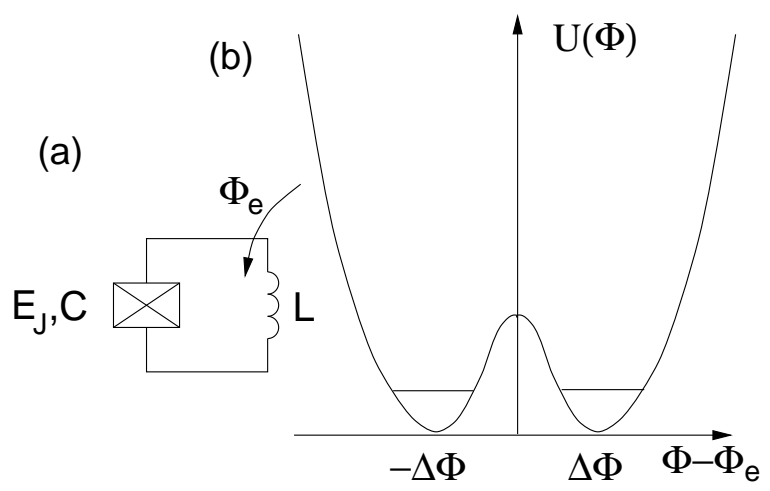

FIG. 6. Equivalent circuit (a) and the energy diagram (b) of the flux qubit. External flux $\Phi_{e}$ induces the flux through the inductance $L$ of the $\operatorname{rf}$ SQUID that is close to half a flux quantum.

The basic scheme of the flux qubit $33-35$ (Fig. 6) is similar to the charge qubit. When external flux through an rf SQUID (7) is equal to a half of the magnetic flux quantum $\Phi_{0}$, and $E_{J} \simeq \Phi_{0}^{2} / 2 \pi^{2} L$, the SQUID potential $U(\Phi)$ has two symmetric minima at $\Phi=\Phi_{0} / 2 \pm \Delta \Phi$ with the two degenerate ground states in them. These two states are separated from the excited states by large energy gaps on the order of quantum of oscillation frequency $\omega_{p}$ around the minima, $\hbar \omega_{p} \simeq\left(E_{J} E_{C}\right)^{1 / 2}$, and are coupled by tunneling through the barrier separating the minima. The tunneling amplitude $\Delta / 2$ is small, $\Delta / 2 \ll \hbar \omega_{p}$. In this regime, the SQUID dynamics reduces to the twostates dynamics at temperatures $T \ll \hbar \omega_{p}$. The energy difference $\varepsilon$ between the two flux states is controlled by the deviations of the external flux $\Phi_{e}$ from $\Phi_{0} / 2$, $\varepsilon \simeq\left(\Phi_{e}-\Phi_{0} / 2\right) \Delta \Phi / L$, while the tunneling amplitude can be controlled by additipmal flux modulating the Josephson coupling energy $E_{J} 25$. This means that if the rate of the environment-induced decoherence of the flux states is suppressed significantly below the tunneling frequency $\Delta$, as in experiments 910 , the rf SQUID can be used as the flux qubit.

After individual qubits, the next step in the design of the quantum computer is the organization of the qubit dynamics necessary to perform quantum logic operations. The main general requirement to this dynamics is that it should maintain the level of precision sufficient for quantum computation. For instance, the amplitudes of the unwanted transitions to the higher-energy states of the system (leading to leakage of the probability out of the two-state qubit space 38 and destroying correct operation of the qubit) should be low. The best and probably the only type of dynamics that satisfies this requirement in the solid-state systems is the adiabatic dynamics 31 . When all qubit parameters are changed adiabatically, the amplitudes of all unwanted transitions decrease exponentially with the ratio of the characteristic qubit energies ( $E_{J}$ for the charge and $\Delta$ for the flux qubits) to the frequency of qubit operation. For comparison, the amplitude of the unwanted transitions in the regime of Rabi oscillatipns employed in the ion-trap 36 or NMR qubits see, e.g. 37 , decreases only linearly with the operation frequency/energy ratio. This means that despite the term "adiabatic", for a given level of accuracy and given qubit energies, adiabatic manipulations of the qubit states can be performed considerably faster than Rabi transitions. Larger operation frequency of adiabatic quantum logic gates is particularly important for solid-state gates which should have significantly shorter decoherence time that the NMR or ion-trap gates.

One more difficult problem of the organization of the quantum logic circuits out of individual qubits is the necessity to switch on and off the interaction between the qubits in the controlled manner. Parameters of the direct Coulomb interaction between the charge qubits and magnetic interaction between the flux qubits typically depend on the system geometry and can not be controlled by external voltage or current signals. Two possible solutions of this problem has been suggested so far. One is the spatial separation of the qubit states in the arrays of junctions 31 , another is making the interaction between the qubits dependent on the "internal" qubit parameter and controlling this parameter 39 . The advantage of the first approach is that it gives the possibility of controlling the interaction with sufficiently high accuracy, as needed for quantum computation. However, the difficulty of fabricating and controlling long arrays of junctions make simpler approaches like 39 attractive for the near-term experiments.

\section{SINGLE-ELECTRON TRANSISTOR AS QUANTUM DETECTOR}

Essential part of the operation of a quantum circuit is the measurement process. In the case of the quantum computing circuits, the measurement is necessary for the read-out of the final state, for error-correction during operation, and, possibly, for preparation of the initial state. The standard detector for measurements of charge in sys- 
tems of mesoscopic tunnel junctions is the single-electron tunneling (SET) transistor 4042 . Over the last ten years, it has been extensively developed $43-48$ and used in many experiments as electrometer - see, e.g. 30.49.50. In this Section, we study the detector properties of SET transistor in a quantum measurement. Quantum measurements with the standard flux detector, a dc SQUID, are discussed in 51 .

SET transistor 40 is a small conductor, i.e., semiconductor quantum dot or small metallic island, placed between two external electrodes (Fig. 7). The conductor forms two tunnel junctions with the electrodes and acts as the central electrode of the transistor. Due to Coulomb charging of this electrode by tunneling electrons, the current $I$ through the junctions can be controlled by the electrostatic potential $\phi$ of the central electrode. Sensitivity of the current $I$ to variations of $\phi$ makes it possible to detect motion of charges in the vicinity of the transistor, and is the basis for its operation as electrometer. When the bias voltage $V$ across the transistor is smaller than the threshold $V_{t}$ of Coulomb blockade, the tunneling is suppressed by the charging energy that is required to transfer an electron in or out of the central electrode. In this regime, the current $I$ flows through the transistor only by a two-step "macroscopic quantum tunneling" (MQT) process via a virtual intermediate-state below the Coulomb energy barrier (for review, see 22 ), and is much smaller than the current above the Coulomb blockade threshold $V_{t}$.

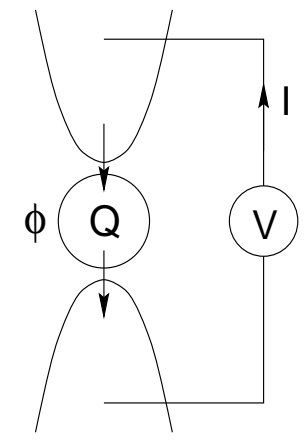

FIG. 7. Diagram of the SET transistor as electrometer. The tunnel current $I$ (measurement output) indicated by arrows is controlled by electrostatic potential $\phi$ of the central electrode of the transistor (input signal) created by a measured system, and is coupled to the transistor through the charge $Q$ of its central electrode.

Rapid current rise at $V \simeq V_{t}$ makes the Coulomb blockade threshold the optimumperating point of the SET transistor as electrometer 5354. Motivated by this fact, we analyze below characteristics of the SET electrometer as a quantum detector close to the Coulomb blockade threshold. Transport properties of the SET transistor in the vicinity of the threshold are determined by the crossover from MQT to the regular single-electron tunneling. The dominant feature of this crossover is quantum broadening of the transistor charge states by tunneling 5355 58. Such a broadening determines the shape of the current-voltage characteristics $I(V)$ at $V \simeq$ $V_{t}$, and can be described in the simplest way in the regime when the temperature and "bias energy" $\mathrm{eV}$ are smaller than the energy spacing of the single-particle states of the central electrode. The tunnel current $I$ flows then only through one single-particle state and the problem becomes equivalent to the standard problem of resonant tunneling through an individual single-particle level. Although this is not the regime of operation of a typical Albased SET electrometer, it is routinely realized in traps port through semiconductor quantum dots - see, e.g.,59, and is reached even in $\mathrm{Al}$ transistor $4 \mathrm{~s}$.

The resonant tunneling through one single-particle level can be described quantitatively with the standard Hamiltonian:

$$
H=\varepsilon a^{\dagger} a+\sum_{j p}\left(\varepsilon_{p} c_{j p}^{\dagger} c_{j p}+T_{j p} c_{j p}^{\dagger} a+T_{j p}^{*} a^{\dagger} c_{j p}\right) .
$$

The operators $c_{j p}$ represent electrons in the state $p$ with energy $\varepsilon_{p}$ of the $j$ th electrode of the transistor, $j=1,2$, while operator $a$ represents electron occupying the resonant level. The energy $\varepsilon$ of the resonant level depends on the electrostatic potential $\phi$ of the central electrode of the transistor, $\delta \varepsilon=-e \delta \phi$. Potential $\phi$ controls the tunnel current $I$ through the transistor and serves as the input signal of the measurement. We assume that the measured system is coupled weakly to the transistor, so that the typical variations of $\phi$ and $\varepsilon$ are small and transistor operates as a linear detector. In the resonant-tunneling regime this implies that $\delta \varepsilon \ll e V$, $\gamma$, where $\gamma$ is the tunneling width of the resonant level. We also assume that the signal frequency is much smaller than $\mathrm{eV}$ and $\gamma$ and treat the transistors response as instantaneous.

The characteristics of a linear detector relevant for quantum measurement are the output noise, back-action noise of the detector on the measured system, and the linear response coefficient of transformation of the input into the output signal - see,e.g.,51. For the SET transistor, the measurement output is the current $I$ through the junctions. The output noise is then the current noise with spectral density $S_{I}(\omega)$, while the response coefficient $\lambda$ is equal to $\delta\langle I\rangle / \delta \phi$, where $\delta\langle I\rangle$ is the change of the average current $I$ due to variation $\delta \phi$ of the potential $\phi$. When the potential $\phi$ is produced by a dynamic system, e.g., a qubit, the transistor is coupled to this system by coupling energy $\phi Q$, where $Q=e a^{\dagger} a$ is the operator of the charge on the resonant level. Since the charge $Q$ fluctuates due to the current flow through the resonant level, the coupling creates a fluctuating force acting on the measured system that leads to dephasing of the quantum states of this system. Such a "backaction dephasing" is the fundamental aspect of a quantum measurement that is responsible for localization of the measured system in the eigenstates of the measured observable. In the case of SET transistor, the measured observable is the potential $\phi$, and intensity of the back- 
action dephasing is determined by the spectral density of the charge noise $S_{Q}(\omega)$.

In the assumed regime of low signal frequency, only the zero-frequency values of the spectral densities of both the output and the input noise, as well as the spectral density $S_{I Q}(\omega)$ of the correlator between $I$ and $Q$ are relevant for the measurement problem. The zero-frequency value of $S_{I Q}(\omega)$ can be calculated as

$$
S_{I Q}=\frac{1}{2 \pi} \int d t[\langle I(t) Q\rangle-\langle I\rangle\langle Q\rangle],
$$

with the zero-frequency limits of $S_{I}(\omega)$ and $S_{Q}(\omega)$ given by similar expressions.

Because of the simple quadratic form of the Hamiltonian (19) all these characteristics of the transistor can be found exactly. Partially, this was done in the theory of charge sensitivity gf the SET electrometer in the resonant-tunneling regime 6 ,61. Calculation of the charge sensitivity for measurements of static charges requires only the knowledge of the current noise $S_{I}$, whereas under a generic measurement conditions one needs to know also the backaction noise $S_{Q}$ and the correlation $S_{I Q}$. Below all spectral densities are calculated following Ref. 0 . In contrast to other treatments $\mathrm{f}$ noise properties of the resonant tunneling structures 62.63 which focus on the suppression of shot noise, discussion of the optimum detector properties of such structures requires the consideration of the noise properties at temperatures and bias voltages smaller than the width $\gamma$ of the resonant level.

The Heisenberg equations of motion for operators $c_{j p}$ and $a$ obtained from the Hamiltonian (19) are:

$$
i \dot{c}_{j p}=\varepsilon_{p} c_{j p}-T_{j p} a, \quad i \dot{a}=\varepsilon a-\sum_{j p} T_{j p}^{*} c_{j p} .
$$

Integrating the first of eqs. (21) we get:

$$
c_{j p}(t)=\bar{c}_{j p}(t)+i T_{j p} \int_{-\infty}^{t} d t^{\prime} e^{-i \varepsilon_{p}\left(t-t^{\prime}\right)} a\left(t^{\prime}\right),
$$

where $\bar{c}_{j p}$ is the "initial value" of $c_{j p}$ that describes the equilibrium occupation of electron states in the transistor electrodes:

$$
\left\langle\bar{c}_{j p}^{\dagger}(t) \bar{c}_{j p}\left(t^{\prime}\right)\right\rangle=f_{j}\left(\varepsilon_{p}\right) e^{i \varepsilon_{p}\left(t-t^{\prime}\right)} .
$$

Here $f_{j}\left(\varepsilon_{p}\right)$ are the Fermi distribution functions with the chemical potentials $\mu_{j}$ of the two electrodes, $\mu_{1}-\mu_{2}=$ $e V>0$.

The second of eqs. (21) combined with eq. (22) can be transformed as

$\dot{a}=-i \varepsilon a+i \sum_{j p} T_{j p}^{*} \bar{c}_{j p}-\sum_{j p}\left|T_{j p}\right|^{2} \int_{0}^{\infty} d t^{\prime} e^{i \varepsilon_{p} t^{\prime}} a\left(t-t^{\prime}\right)$.

Under the assumption that the electrodes have continuous spectrum of energy states, and that the densities of states $\rho_{j}\left(\varepsilon_{p}\right)$ together with the matrix elements of tunneling are constant in a wide energy range around $\varepsilon_{p} \simeq \varepsilon$, the last term in this equation reduces to $-\gamma a(t)$, with

$$
\gamma=\gamma_{1}+\gamma_{2}, \quad \gamma_{j}=\pi\left|T_{j}\right|^{2} \rho_{j}(\varepsilon) .
$$

Here $\left|T_{j}\right|^{2}$ is the tunneling probability $\left|T_{j p}\right|^{2}$ averaged over all states with the same energy $\varepsilon_{p}$, and $\gamma_{j}$ is half of the tunneling rate between the $j$ th electrode and the resonant level. After this transformation, eq. (24) gives:

$$
a(t)=\sum_{j p} \frac{T_{j p}^{*} \bar{c}_{j p}(t)}{\varepsilon-\varepsilon_{p}-i \gamma} .
$$

Equation (25) allows us to find the backaction noise $S_{Q}$ :

$$
\begin{gathered}
S_{Q}=\frac{e^{2}}{2 \pi} \int d t\left\langle a^{\dagger}(t) a\right\rangle\left\langle a(t) a^{\dagger}\right\rangle= \\
\frac{e^{2}}{\pi^{2}} \sum_{i, j} \gamma_{i} \gamma_{j} \int \frac{d \nu}{\left[(\varepsilon-\nu)^{2}+\gamma^{2}\right]^{2}} f_{i}(\nu)\left(1-f_{j}(\nu)\right) .
\end{gathered}
$$

In the limit of low temperatures, $T \ll e V$, $\gamma$, relevant for transistor operation as a quantum detector, eq. (26) reduces to the following form:

$$
S_{Q}=\frac{e^{2} \gamma_{1} \gamma_{2}}{\pi^{2}} \int_{\mu_{2}}^{\mu_{1}} \frac{d \nu}{\left[(\varepsilon-\nu)^{2}+\gamma^{2}\right]^{2}} .
$$

When either temperature $T$ or bias $e V$ are larger than the tunneling width $\gamma$, integral in eq. (26) can be taken treating $f$ 's as constants, and gives expression for the noise $S_{Q}$ that can also be obtained from solution of a simple kinetic equation for the occupation probabilities of the resonant level. The same conclusion applies to eq. (27) in the case of large voltages, $\mathrm{eV} \gg \gamma$, when the noise $S_{Q}$ in the occupation of the resonant level is due the shot noise of the tunneling current. In this case, eq. (27) gives:

$$
S_{Q}=\frac{e^{2} \gamma_{1} \gamma_{2}}{2 \pi \gamma^{3}} .
$$

In order to find the average current $\langle I\rangle$ and the noise $S_{I}$ at low frequencies, the tunneling current $I$ through the transistor can be calculated in either of the junctions. In the first junction,

$$
I=i e \sum_{p}\left(T_{1 p} c_{1 p}^{\dagger} a-T_{1 p}^{*} a^{\dagger} c_{1 p}\right) .
$$

Using explicit expression (25) for $a$ in eq. (22) to express $c_{j p}$ in terms of $\bar{c}_{j p}$, we write the operator (29) of the current $I$ as

$$
I=i e\left(A^{\dagger} a-a^{\dagger} A\right)
$$

where 


$$
A^{\dagger} \equiv \sum_{p} T_{1 p} \bar{c}_{1 p}^{\dagger}-i \gamma_{1} \sum_{j p} \frac{T_{j p} \bar{c}_{j p}^{\dagger}(t)}{\varepsilon-\varepsilon_{p}+i \gamma} .
$$

Averaging eq. (30) over the equilibrium state of the electrodes as in the eq. (23), we get the standard result for the dc current through a resonant level, and find the response coefficient $\lambda \equiv \delta\langle I\rangle / \delta \phi$ :

$$
\lambda=\frac{4 e^{2} \gamma_{1} \gamma_{2}}{\pi} \int d \nu \frac{\varepsilon-\nu}{\left[(\varepsilon-\nu)^{2}+\gamma^{2}\right]^{2}}\left(f_{1}(\nu)-f_{2}(\nu)\right) .
$$

At large bias, when $\varepsilon-\mu_{2} \gg \gamma$ and $E \equiv \mu_{1}-\varepsilon \gg \gamma$, the response coefficient decreases, e.g., for low temperatures,

$$
\lambda=\frac{2 e^{2} \gamma_{1} \gamma_{2}}{\pi E^{2}} .
$$

The noise $S_{I}$ can also be found from eq. (30). Similarly to eqs. (20) and (26), we get:

$$
\begin{gathered}
S_{I}=\frac{e^{2}}{2 \pi} \int d t\left[\left\langle A^{\dagger}(t) A\right\rangle\left\langle a(t) a^{\dagger}\right\rangle+\left\langle A(t) A^{\dagger}\right\rangle\left\langle a^{\dagger}(t) a\right\rangle-\right. \\
\left.\left\langle A^{\dagger}(t) a\right\rangle\left\langle a(t) A^{\dagger}\right\rangle-\left\langle A(t) A^{\dagger}\right\rangle\left\langle a^{\dagger}(t) a\right\rangle\right] .
\end{gathered}
$$

Evaluating averages in this expression with the help of eqs. (31) and (25), we get after some algebra:

$$
\begin{gathered}
S_{I}=\frac{e^{2} \gamma_{1} \gamma_{2}}{\pi^{2}} \int \frac{d \nu}{\left[(\varepsilon-\nu)^{2}+\gamma^{2}\right]^{2}} \\
{\left[\gamma_{1} \gamma_{2}\left(f_{1}(\nu)\left(1-f_{1}(\nu)\right)+f_{2}(\nu)\left(1-f_{2}(\nu)\right)\right)+\right.} \\
\left.\left((\varepsilon-\nu)^{2}+\left(\gamma_{1}-\gamma_{2}\right)^{2}\right)\left(f_{1}(\nu)\left(1-f_{2}(\nu)\right)+\left(1-f_{1}(\nu)\right) f_{2}(\nu)\right)\right] .
\end{gathered}
$$

Equation (34) gives the current noise at arbitrary temperatures and bias voltages. In the limit of small temperatures, it can be written as

$$
\begin{gathered}
S_{I}=\int_{\mu_{2}}^{\mu_{1}} d \nu S_{I}(\nu), \\
S_{I}(\nu)=\frac{e^{2} \gamma_{1} \gamma_{2}}{\pi^{2}} \frac{(\varepsilon-\nu)^{2}+\left(\gamma_{1}-\gamma_{2}\right)^{2}}{\left[(\varepsilon-\nu)^{2}+\gamma^{2}\right]^{2}} .
\end{gathered}
$$

Note that the partial contribution $S_{I}(\nu) d \nu$ to the current noise (35) from an energy interval $d \nu$ around some energy $\nu$ is given by the standard general equation for the noise of a mesoscopic conductor - see, e.g. $64, S_{I}(\nu) d \nu=$ $\left(e^{2} d \nu / 4 \pi^{2}\right) D(1-D)$, where the transparency $D$ of the resonant-tunneling structure is energy-dependent: $D=$ $4 \gamma_{1} \gamma_{2} /\left((\varepsilon-\nu)^{2}+\gamma^{2}\right)$, and the width $d \nu$ of the energy interval acts as the bias energy $\mathrm{eV}$.

In the large-bias limit, eq. (35) describes current noise associated with the inceherent sequential tunneling through the resonant level62,63,60:

$$
S_{I}=\frac{e\langle I\rangle}{2 \pi}\left(1-\frac{2 \gamma_{1} \gamma_{2}}{\gamma^{2}}\right) .
$$

where the average current through the level is $\langle I\rangle=$ $2 e \gamma_{1} \gamma_{2} / \gamma$. In structures that are not highly asymmetric, $\gamma_{1} \sim \gamma_{2}$, eq. (36) describes suppression of the current noise in comparison with the regular shot noise $e\langle I\rangle / 2 \pi$. The previous discussion shows that this suppression has two correct interpretations that appear to be quite different. One explanation relates the suppression to the quantum Fermi correlations in the tunneling flow of electrons through the structure described by the transparency-dependent suppression factor $(1-D)$. In another interpretation, the suppression is brought about by the classical correlations in the process of electron transfer through the structure, which break this process into two distinct steps, tunneling onto the resonant level and out of it.

Finally, following the same steps that lead to eq. (34) for the current noise, we calculate the current-charge correlator $S_{I Q}$. From eqs. (20) and (30), and the definition of $Q$ we have:

$$
S_{I Q}=\frac{i e^{2}}{2 \pi} \int d t\left[\left\langle A^{\dagger}(t) a\right\rangle\left\langle a(t) a^{\dagger}\right\rangle-\left\langle A(t) a^{\dagger}\right\rangle\left\langle a^{\dagger}(t) a\right\rangle\right] .
$$

Making use of eqs. (25) and (31) to calculate the averages in this expression we obtain:

$$
\begin{gathered}
S_{I Q}=\frac{e^{2} \gamma_{1} \gamma_{2}}{\pi^{2}} \int \frac{d \nu}{\left[(\varepsilon-\nu)^{2}+\gamma^{2}\right]^{2}}\left[\gamma_{1} f_{1}(\nu)\left(1-f_{1}(\nu)\right)-\right. \\
\gamma_{2} f_{2}(\nu)\left(1-f_{2}(\nu)\right)+\left(\gamma_{2}-\gamma_{1}-i(\varepsilon-\nu)\right) f_{1}(\nu)\left(1-f_{2}(\nu)\right)+ \\
\left.\left.\left(\gamma_{2}-\gamma_{1}+i(\varepsilon-\nu)\right)\left(1-f_{1}(\nu)\right) f_{2}(\nu)\right)\right] .
\end{gathered}
$$

At $T \ll \gamma, e V$, this equation reduces to

$$
S_{I Q}=\frac{e^{2} \gamma_{1} \gamma_{2}}{\pi^{2}} \int_{\mu_{2}}^{\mu_{1}} d \nu \frac{\gamma_{2}-\gamma_{1}-i(\varepsilon-\nu)}{\left[(\varepsilon-\nu)^{2}+\gamma^{2}\right]^{2}} .
$$

Comparing eqs. (27), (35), and (38) for the lowtemperature noise spectral densities, and the lowtemperature limit of eq. (32) for the response coefficient, we can see that the partial contributions to these functions from tunneling of electrons with a certain energy $\nu$ satisfy the generic relations of a linear quantum-limited detector 1 :

$$
S_{I}(\nu) S_{Q}(\nu)=\left|S_{I Q}(\nu)\right|^{2}, \quad \operatorname{Im} S_{I Q}(\nu)=-\lambda(\nu) / 4 \pi,
$$

while, similarly to other types of detectors, $\operatorname{Re} S_{I Q}$ characterizes the detector asymmetry. At small bias voltages, $\mathrm{eV} \ll \gamma$, when the tunnel current is determined by tunneling in the narrow energy range, the actual values of $\lambda$ and all spectral densities coincide with $\lambda(\nu), S_{I}(\gamma)$, etc. According to generic arguments presented in Ref.[1, 
eqs. (39) mean that the SET transistor in this regime is a quantum-limited detector reaching the fundamental limit of the energy sensitivity $\epsilon$ :

$$
\epsilon \equiv \frac{2 \pi}{\lambda(\nu)}\left[S_{I}(\nu) S_{Q}(\nu)-\left(\operatorname{Re} S_{I Q}(\nu)\right)^{2}\right]^{1 / 2}=\hbar / 2 .
$$

Such a quantum detector also has an ultimate signalto-noise ratio $R$ for a continuous weak measurement of a qubit. If the qubit oscillates between its two basis states, the signal-to-noise ratio can be defined as the ratio of the amplitude of the oscillation line in the output spectrum of the detector to the output noise. The ratio $R$ can be shown 51 to be closely related to the energy sensitivity (40):

$$
R=\frac{\lambda(\nu)^{2}}{4 \pi^{2} S_{I}(\nu) S_{Q}(\nu)} .
$$

For a symmetric detector with $\operatorname{Re} S_{I Q}(\nu)=0$ (i.e., in the case of SET transistor, when $\gamma_{1}=\gamma_{2}$ ), the signal-tonoise ratio $R$ can be written as $(\hbar / \epsilon)^{2}$ and is limited by 4.65 This fundamental signal-to-noise ratio means that the detector does not add more dephasing into the qubit dynamics than the minimum required by the afuisition of information about the state of the system 66,51

The small bias condition necessary for the SET transistor to operate as a quantum-limited detector with the energy sensitivity $\hbar / 2$ limits the absolute value of the transistor output (the current $I$ ). In order to see to what extent the transistor properties are degraded by increasing bias, we consider the case when the "collector" electrode absorbs all tunneling electrons, $\varepsilon-\mu_{2} \gg \gamma$. Detector characteristics of the transistor, most importantly energy sensitivity $\epsilon$, depend then on the position of the resonant energy level relative to the chemical potential of the "emitter" electrode characterized by $E=\mu_{1}-\varepsilon$. Integrating eqs. (27), (35), (38), and (32) we find the noise spectral densities as functions of $E$ :

$$
\begin{gathered}
S_{I}=\frac{e^{2} \gamma_{1} \gamma_{2}}{\pi^{2} \gamma^{3}}\left[\left(\gamma_{1}^{2}+\gamma_{2}^{2}\right)\left(\frac{\pi}{2}+\tan ^{-1} z\right)-2 \gamma_{1} \gamma_{2} \frac{z}{1+z^{2}}\right], \\
S_{Q}=\frac{e^{2} \gamma_{1} \gamma_{2}}{\pi^{2} \gamma^{3}}\left[\frac{\pi}{2}+\tan ^{-1} z+\frac{z}{1+z^{2}}\right], \quad z \equiv E / \gamma, \quad(41) \\
\operatorname{Re}_{I Q}=\left(\gamma_{1}-\gamma_{2}\right) S_{Q}, \quad-\operatorname{Im} S_{I Q}=\frac{\lambda}{4 \pi}=\frac{e^{2} \gamma_{1} \gamma_{2}}{2 \pi^{2} \gamma^{2}} \frac{1}{1+z^{2}} .
\end{gathered}
$$

Using eqs. (41) we obtain that the energy sensitivity $\epsilon=2 \pi\left[S_{I} S_{Q}-\left(\operatorname{Re} S_{I Q}\right)^{2}\right]^{1 / 2} / \lambda$ of SET transistor in the resonant-tunneling regime is independent of the degree of the transistor asymmetry $\gamma_{1} / \gamma_{2}$ :

$$
\epsilon=\frac{\hbar}{2}\left[\left(1+z^{2}\right)^{2}\left(\frac{\pi}{2}+\tan ^{-1} z\right)^{2}-z^{2}\right]^{1 / 2} .
$$

Equation 42 is plotted in Fig. 8. We see that the behavior of $\epsilon$ as a function of $E$ changes from being nearlyconstant for $E<0$ to rapid increase with $E$ at $E>0$. This rapid increase reflects suppression of the response coefficient $\lambda(33)$ with increasing bias. Quantitatively, eq. (42) gives for $|E| \gg \gamma$ :

$$
\epsilon=\hbar\left\{\begin{array}{l}
\pi E^{2} / 2 \gamma^{2}, \quad E>0 \\
1 / \sqrt{3}, \quad E<0
\end{array}\right.
$$

This equation shows that in the regime of quantum tunneling, $E<0$, the energy sensitivity comes very close to the fundamental limit $\hbar / 2$. It does not quite reach it because of the energy dependence of the tunneling rate.

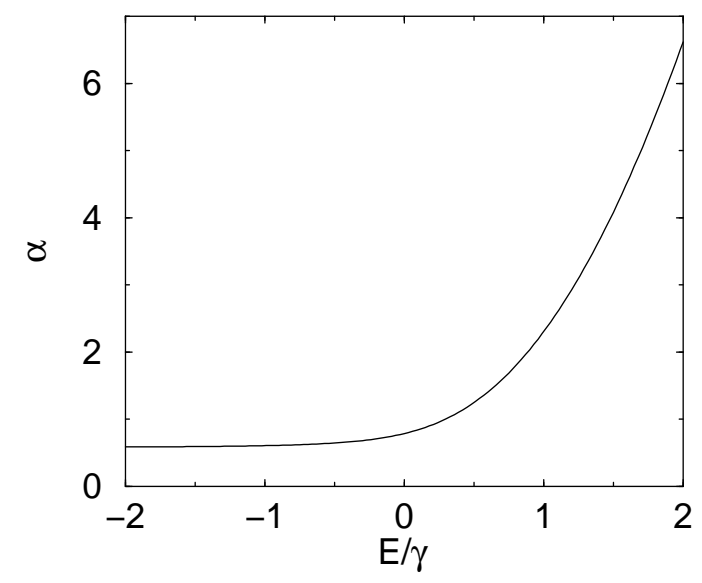

FIG. 8. Low-temperature energy sensitivity (42) of the SET transistor in the resonant-tunneling regime in units of $\hbar, \alpha \equiv \epsilon / \hbar$, as a function of the bias energy $E$ characterizing position of the resonant level relative to the chemical potential of the emitter electrode.

Figure 9 shows the signal-to-noise ratio $R=$ $\lambda^{2} /\left(4 \pi^{2} S_{I} S_{Q}\right)$ for continuous measurement of a qubit using the SET transistor in the resonant-tunneling regime that is calculated from eqs. (41). In contrast to $\epsilon, R$ depends on the transistor asymmetry, but the magnitude of variation of $R$ with $\gamma_{1} / \gamma_{2}$ is small, and regardless of $\gamma_{1} / \gamma_{2}$, the signal-to-noise ratio approaches 3 at large negative $E$. In the symmetric case, $\gamma_{1} / \gamma_{2}=1$, the signalto-noise ratio is given by the general relation $R=(\hbar / \epsilon)^{2}$ with energy sensitivity (42).

To conclude, we see that the SET transistor in the resonant-tunneling regime biased at the Coulomb blockade threshold should operate at low temperatures as a quantum-limited detector. It has energy sensitivity $\hbar / \sqrt{3}$ and signal-to-noise ratio 3 for continuous weak measurement of a qubit. In this regime, transistor introduces the noise in the dynamics of the measured system that is only marginally larger than the fundamental noise required by the quantum mechanics of measurement. This means that for the optimally-biased transistor the time of the backaction dephasing is equal to the measurement time. This conclusion differs from the results of the previqus analysis of the SET transistor at large bias voltages67, 
where the transistor generates strong shot noise leading to the backaction dephasing that is much stronger than the fundamental minimum.

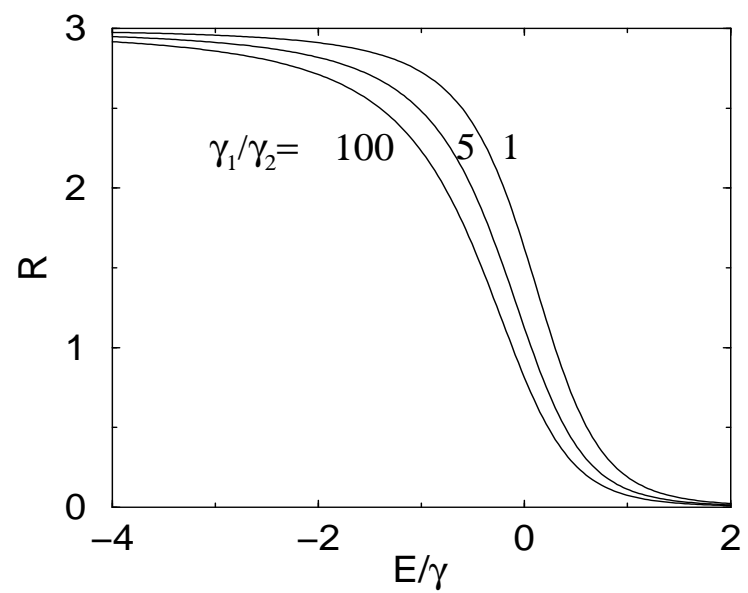

FIG. 9. Signal-to-noise ratio $R$ for continuous weak measurement of a qubit with the SET transistor in the resonant-tunneling regime. $R$ is shown as a function of the bias energy $E$ for various degrees of the transistor asymmetry.

The author would like to acknowledge useful discussions with A.N. Korotkov. This work was supported by $\mathrm{ARO}$ and AFOSR.

${ }^{1}$ P.M.A. Dirac, Proc. R. Soc. A 114, 243 (1927).

${ }^{2}$ P.W. Anderson, in: "The many-body problem", ed. by E.R. Caianiello, (Academic Press, New York, 1964), vol. 2, p. 113.

3 "Quantum tunneling in condensed media", ed. by Yu. Kagan and A.J. Leggettt, (North-Holland, Amsterdam, 1992).

${ }^{4}$ D.V. Averin and K.K. Likharev, in: Mesoscopic Phenomena in Solids, ed. by B.L. Altshuler, P.A. Lee, and R.A. Webb, (Elsevier, Amsterdam, 1991), p. 173.

${ }^{5}$ G. Schön and A.D. Zaikin, Phys. Rep. 198, 237 (1990).

6 "Single Charge Tunneling", ed. by H. Grabert and M. Devoret (Plenum, NY, 1992).

${ }^{7}$ D. Loss and D.P. DiVincenzo, Phys. Rev. A 57, 120 (1998).

${ }^{8}$ Y. Nakamura, Yu.A. Pashkin, and J.S. Tsai, Nature 398, 786 (1999).

9 J.R. Friedman, V. Patel, W. Chen, S.K. Tolpygo, and J.E. Lukens, cond-mat/0004293.

${ }^{10}$ J.E. Mooij, private communication.

${ }^{11}$ D.V. Averin, A.B. Zorin, and K.K. Likharev, Zh. Eksp. Teor. Fiz. 88, 692 (1985) [Sov. Phys. JETP 61, 407].

12 D.T. Pegg and S.M. Barnett, J. Mod. Optics 44, 225 (1997).

13 P.W. Anderson, J. Phys. Chem. Solids 11, 28 (1959).

${ }^{14}$ K.A. Matveev and A.I. Larkin, Phys. Rev. Lett. 78, 3749 (1997).
15 P.G. de Gennes, "Superconductivity of Metals and Alloys" (Addison-Wesley, 1989).

${ }^{16}$ W. Zerger, A.T. Dorsey, and M.P.A. Fisher, Phys. Rev. B, 34, 6518 (1986).

17 S.M. Apenko, Phys. Lett. A 142, 277 (1989).

18 D. Loss and K. Mullen, Phys. Rev. A 43, 2129 (1991).

${ }^{19}$ Y.Nakamura, C.D. Chen, and J.S. Tsai, Phys. Rev. Lett. 79, 2328 (1997).

${ }^{20}$ V. Bouchiat, D. Vion, P. Joyez, D. Esteve, and M.H. Devoret, J. of Supercond. 12, 789 (1999).

${ }^{21}$ D.J. Flees, S. Han, and J.E. Lukens, J. of Supercond. 12, 813 (1999).

${ }^{22}$ D.V. Averin, J.R. Friedman, and J.E. Lukens, condmat/005081.

${ }^{23}$ D.V. Averin, J. Low Temp. Phys. 118, 781 (2000).

${ }^{24}$ Yu.N. Ovchinnikov and A. Schmidt, Phys. Rev. B 50, 6332 (1994).

${ }^{25}$ R. Rouse, S. Han, and J.E. Lukens, Phys. Rev. Lett. 75, 1614 (1995).

${ }^{26}$ P. Silvestrini, V.G. Palmieri, B. Ruggiero, and M. Russo, Phys. Rev. Lett. 79, 3046 (1997).

${ }^{27}$ K. Blum, "Density matrix theory and applications", (Plenum, NY, 1981).

${ }^{28}$ M. Büttiker, Phys. Rev. B 36, 3548 (1987).

${ }^{29}$ P. Lafarge, H. Pothier, E.R. Williams, D. Esteve, C. Urbina, and M.H. Devoret, Z. Phys. B 85, 327 (1991).

${ }^{30}$ P. Lafarge, P. Joyes, D. Esteve, C. Urbina, and M.H. Devoret, Phys. Rev. Lett 70, 994 (1993).

${ }^{31}$ D.V. Averin, Solid State Commun. 105, 659 (1998).

${ }^{32}$ Yu. Makhlin, G. Schön, and A. Shnirman, Nature 398, 305 (1999).

${ }^{33}$ L.B. Ioffe, V.B. Geshkenbein, M.V. Feigel'man, A.L. Fauchère, and G. Blatter, Nature 398, 679 (1999).

34 J.E. Mooij, T.P. Orlando, L. Levitov, Lin Tian, Caspar H. van der Wal, and Seth Lloyd, Science 285, 1036 (1999).

35 A. Blais and A. M. Zagoskin, Phys. Rev. A, 61042308 (2000).

${ }^{36}$ C. Monroe, D.M. Meekhof, B.E. King, W.M. Itano, and D.J. Wineland, Phys. Rev. Lett. 75, 4714 (1995).

${ }^{37}$ I.L. Chuang, N.A. Gershenfeld, and M. Kubinec, Phys. Rev. Lett. 80, 3408 (1998).

${ }^{38}$ R. Fazio, G.M. Palma, and J. Siewert, Phys. Rev. Lett. 83, 5385 (1999).

${ }^{39}$ Yu. Makhlin, G. Schön, and A. Shnirman, J. Low Temp. Phys. 118, 751 (2000).

${ }^{40}$ D.V. Averin and K.K. Likharev, J. Low Temp. Phys. 62, 345 (1986).

41 T.A. Fulton and G.J. Dolan, Phys. Rev. Lett 59, 109 (1987).

42 K.K. Likharev, IEEE Trans. Magn. 23, 1142 (1987).

${ }^{43}$ E.H. Visscher, S.M. Verbrugh, J. Lindeman, P. Hadley, and J.E. Mooij, Appl. Phys. Lett. 66, 305 (1995).

${ }^{44}$ M.J. Yoo, T.A. Fulton, H.F. Hess, R.L. Willett, L.N. Dunkleberger, R.J. Chichester, L.N. Pfeiffer, and K.W. West, Science 276, 579 (1997).

${ }^{45}$ R.J. Schoelkopf, P. Wahlgren, A.A. Kozhevnikov, P. Delsing, and D.E. Prober, Science 280, 1238 (1998).

${ }^{46}$ B. Starmark, T. Henning, T. Claeson, P. Delsing, and A.N. Korotkov, J. Appl. Phys. 86, 2132 (1999).

${ }^{47}$ V.A. Krupenin, D.E. Presnov, A.B. Zorin, and J. Niemeyer, 
J. Low Temp. Phys. 118, 287 (2000).

${ }^{48}$ Yu.A. Pashkin, Y. Nakamura, and J.S. Tsai, Appl. Phys. Lett. 76, 2256 (2000).

${ }^{49}$ P.D. Dresselhaus, L. Ji, S. Han, J.E. Lukens, and K.K. Likharev, Phys. Rev. Lett 72, 3226 (1994).

${ }^{50}$ Y. Y. Wei, J. Weis, K. v. Klitzing, and K. Eberl, Phys. Rev. Lett 81, 1674 (1998).

${ }^{51}$ D.V. Averin, in: "Exploring the Quantum-Classical Frontier: Recent Advances in Macroscopic and Mesoscopic Quantum Phenomena", Eds. J.R. Friedman and S. Han, to be published; cond-mat/0004364.

${ }^{52}$ D.V. Averin and Yu.V. Nazarov, in Ref6, p. 217.

${ }^{53}$ A.N. Korotkov, D.V. Averin, K.K. Likharev, and S.A. Vasenko, in: "Single Electron Tunneling and Mesoscopic devices", Ed. by H. Koch and H. Lübbig, (Springer, Berlin, 1992), p. 45.

${ }^{54}$ U. Hanke, Yu.M. Galperin, and K.A. Chao, Appl. Phys. Lett. 65, 1847 (1994); Erratum 65, 3416 (1994).

${ }^{55}$ Yu.V. Nazarov, J. Low Temp. Phys. 90, 77 (1993).

${ }^{56}$ P. Lafarge and D. Esteve, Phys. Rev. B 48, 14309 (1993).

${ }^{57}$ D.V. Averin, Physica B, 194-196, 979 (1994).

${ }^{58}$ H. Schoeller and G. Schön, Phys. Rev. B 50, 18436 (1994).

${ }^{59}$ L.P. Kouwenhoven, C.M. Marcus, P.L. McEuen, S. Tarucha, R.M. Westervelt, and N.S. Wingreen, in: "Mesoscopic Electron Transport", Ed. by L.L. Sohn, L.P. Kouwenhoven, and G. Schön, (Kluwer, 1997), p. 105.

${ }^{60}$ D.V. Averin, J. Appl. Phys. 73, 2593 (1923).

${ }^{61}$ The charge sensitivity discussed in Ref.00 was expressed through the energy sensitivity $\delta \varepsilon$ of the tunnel current to variations of the position of the resonant level. Note that this energy sensitivity is different from the more standard energy sensitivity $\epsilon$ (40) studied in this work.

${ }^{62}$ L.Y. Chen and C.S. Ting, Phys. Rev. B 43, 4534 (1991).

${ }^{63}$ J.H. Davies, P. Hyldgaard, S. Hershfield, and J.W. Wilkins, Phys. Rev. B 46, 9620 (1992).

${ }^{64}$ Ya.M. Blanter and M. Buttiker, cond-mat/9910158.

${ }^{65}$ A.N. Korotkov and D.V. Averin, cond-mat/0002203

${ }^{66}$ A.N. Korotkov, Phys. Rev. B 60, 5737 (1999).

${ }^{67}$ A. Shnirman and G. Schön, Phys. Rev. B 57, 15400 (1998). 